\title{
Advancing best practices in data analysis with automatic and optimized output data analysis software
}

\author{
Attila Krajcsi \\ Department of Cognitive Psychology, Institute of Psychology, ELTE Eötvös Loránd University
}

Current data analysis practice and statistics education are suboptimal in many senses, which contributes to the replication crisis. To address some of these issues, a new type of statistical and data analysis software solution is proposed here in which most of the analysis steps are compiled automatically based on the task and the measurement level of the variables and in which the result output is carefully optimized for informativeness and understandability. Automatic data analysis and optimized output can contribute to making data analysis more coherent across studies, remedying some aspects of the issues leading to the replication crisis, making analysis more efficient for users and helping to promote and teach better data analysis practices. Such a solution can be useful for researchers to conduct faster and more precise analyses, for students to see illustrations and demonstrations of data analysis solutions, and for methodologists to formulate straightforward analysis procedures that can promote more precise and more coherent data analysis practice in the literature. A possible implementation of such software, CogStat is presented here, in which additional design considerations make the results more understandable and more precise, the analyses more accessible, and the analysis more efficient.

Various challenges exist in the statistical analysis practice and statistics education. In recent years, the replication crisis has garnered considerable attention (Ioannidis, 2005; Open Science Collaboration, 2015), and several works have provided arguments on the potential sources of the crisis (Carp, 2012b; Silberzahn et al., 2018; Simmons et al., 2011; Wicherts, 2017), and many suggestions have been provided to decrease the adverse effects (Cumming, 2014; Maner, 2014; Wagenmakers et al., 2017; Wicherts, 2017; Wicherts et al., 2016). A large portion of the causes and recommendations are related to data processing and statistical analysis practices. Another related issue is the efficiency of statistics education and the appropriateness of the procedures used by researchers (Beyth-Marom et al., 2008; Wicherts, 2017). Moreover, current software solutions are suboptimal in the sense that they require several mechanic steps from users that can be otherwise automatized. 
In this paper, it is argued that automatic data analysis and automatic presentation of appropriate results in software solutions can partly resolve these issues. More appropriate software solutions can decrease several replication-related issues, improve statistics education, and improve the efficiency of users.

The first section of this paper summarizes some of the relevant problems. In the second section, general principles of possible automatic software solutions are presented and discussed. The third section introduces some related principles on how results can be displayed in a more optimized way. The fourth section introduces a possible implementation of these principles in the CogStat software. The fifth section summarizes the main advantages of this type of analysis solution.

\section{Issues in the statistical analysis practice}

One crucial problem that automatic analysis aims to solve is the replication crisis. This crisis has several symptoms. One key symptom is that reported effect sizes are exaggerated: Reported effect sizes are higher than the real effect sizes (Open Science Collaboration, 2015), and, in hypothesis tests, reported results are more frequently significant with smaller p values than the real effects. For example, the number of $\mathrm{p}$ values between 0.05 and 0.045 is higher than expected based on the overall distribution of the p values, which reflects a publication bias (Masicampo \& Lalande, 2012). Relatedly, some reported effects do not exist at all; instead, random noises are interpreted to be existent effects (Open Science Collaboration, 2015).

Various important factors enable or lead to these main symptoms. Notably, data sampling is unavoidably noisy. It is not only the main question of statistical analyses, that is, how existing effects can be identified in the light of unavoidably noisy data, but it is also a considerable challenge for practicing experts to differentiate between real effects and noise. A second source of potential issues is that most of the investigated effects are formed by an interaction of causes. It is important to highlight that, unlike the factors described below, these two factors (i.e., noisy sampling and interacting causes) are unavoidable, and no scientific method can make them disappear. Another critical factor is the bias for positive results: Editors, reviewers, and, consequently, authors prefer positive results over negative results (Nosek et al., 2012; Rosenthal, 1979). Although the distorting effect of this bias has been recognized and, measurements have been initiated to reduce its effect (Nosek et al., 2012; Wicherts, 2017; Wicherts et al., 2016); in practice, the bias for positive results is still dominant. Another essential factor is the diversity of analysis methods for the same tasks combined with a lack of consensus on their use. For example, the normality of a sample can be investigated with several methods, but there is a notable lack of a single recommended method (or even a select few); moreover, even whether to use hypothesis tests as an appropriate solution for a normality assumption check is under debate (Rochon et al., 2012; Schucany \& Tony Ng, 2006; Wilkinson, 1999), not to mention, which hypothesis test is recommended (Farrell \& Rogers-Stewart, 2006; Noughabi \& Arghami, 2011; Romão et al., 2010; Yap \& Sim, 2011). Note that the lack of consensus can be observed on several levels: In some cases, methodological experts do not agree upon a method (e.g., in the normality assumption as mentioned above); in other cases, while experts have formed a consensus, research practice does not adhere to 
this consensus (e.g., the assumption of hypothesis tests are often not verified [Hoekstra et al., 2012]); in other cases, researchers use the analyses that are offered by their default statistical software without considering their methodological issues. Note that the two latter factors (i.e., bias for positive results and lack of consensus), unlike sampling noise and various interacting causes, can be avoided in theory, although current practice is far from resolving them.

In light of this background, researchers currently follow many practices that lead to the replication crisis, and many of these practices and their consequences are well-documented. Scholars tend to hypothesize after the results are known (termed HARKing), which leads to biased results (Kerr, 1998). Researchers frequently select what details or what projects to publish or what variables to use as covariates or outcomes, partly trying to satisfy the expectation of positive results (Rosenthal, 1979; Silberzahn et al., 2018; Wicherts, 2017). There are many optional procedures that can be arbitrarily combined (p-hacking) leading to various results; the researcher will then publish the result closest to their expectation (Simmons et al., 2011; Wicherts et al., 2016). Overall, throughout the analysis process, there are numerous opportunities for researchers to choose from various solutions, wherein justifications for researchers' specific solutions are often not established, leading to unnecessary degrees of freedom in the analysis (Bowring et al., 2019; Carp, 2012a, 2012b; Silberzahn et al., 2018; Simmons et al., 2011; Wicherts et al., 2016).

Some of these issues are related to statistics education and, consequently, whether or not researchers follow the procedure that is recommended in most statistical courses. For example, it has been demonstrated that, in many cases, detailed distribution of the data is not investigated (Weissgerber et al., 2015), assumptions of hypothesis tests are not checked appropriately (Hoekstra et al., 2012), results of hypothesis testing are misreported (Bakker \& Wicherts, 2011), and more generally, hypothesis testing is frequently misunderstood (Cumming, 2014; Nickerson, 2000). Relatedly, some new methods are not widely known, or it is not straightforward which already existing and well-known solution is the best to use. For example, there are more than 40 hypothesis tests for testing the normality of a variable, and this large number makes it more difficult for researchers to know which are recommended to use and which are not. In addition, even if there are existing solutions, the procedure may not be accessible. For example, although there are available methods to compare a single case with a control group (Crawford \& Howell, 1998), these solutions are not used in all appropriate cases.

Many of these nonideal actions by researchers and the consequences on appropriate interpretation of statistical results and on replicability are well-documented in the literature. To attenuate these effects, several proposals have been put forward. For example, preregistration and registered reports substantially reduce HARKing, p-hacking, and selective reporting (Chambers, 2013; Rosenthal, 1979; Wicherts, 2017). Data sharing improves transparency and as a consequence, improves the overall quality of data analysis (Wicherts et al., 2006, 2011). Replications and meta-analyses (including mini meta-analyses [Maner, 2014]) are effective tools against sampling-related noise, phacking, and selective reporting (Carp, 2012b; Open Science Collaboration, 2015; Wicherts, 2017; Wicherts et al., 2006). Regarding misunderstandings about frequentist hypothesis tests, several alternative properties of the effects have been proposed, such as interval estimations (Cumming \& 
Finch, 2005), effect sizes (Fritz et al., 2012), or Bayesian hypothesis tests and other Bayesian solutions (Wagenmakers, 2007; Wagenmakers et al., 2017); see further examples in Nickerson (2000). Relatedly, improving statistics education leads to better data analysis practices (BeythMarom et al., 2008; Wicherts, 2017). Finding consensus on analysis procedures decreases unnecessary degrees of freedom for researchers and makes reported results more comparable (Bowring et al., 2019; Carp, 2012a). Reports that are easier to understand improve the quality of the interpretation of results (Cumming, 2014; Cumming \& Finch, 2005; Killeen, 2005). Many of these recommendations are gaining momentum and leading to improved quality in reported results. Nonetheless, there is a still lot of room for improvement. To further improve quality of reported results, an alternative software solution type is proposed here, in which data are analyzed automatically, and the results are presented in a thoughtfully designed way.

\section{The possibility of automatic data analysis}

Many issues within statistical analysis can be partly resolved with automatic analysis. Automatic analysis can make analyses faster, more precise, and more coherent. The present section describes some key features of automatic data analysis and considers some of its issues.

In an automatic data analysis software, analyses are mostly performed without interaction or direct guidance from the user and are performed based on recommended analysis procedures. For example, in the case of hypothesis tests, there are clear textbook descriptions of which tests are recommended for specific questions, measurement levels, and variables. Moreover, it is clear what assumptions should be fulfilled for the hypothesis tests, and there are various solutions for how these assumptions should be examined. Similarly, there are recommendations for what descriptives are the most relevant for summarizing a variable, what charts are among the most informative for depicting visually the analyses, etc. Based on these considerations, a recommended analysis pipeline can be built based on the analysis question, variables, and their measurement levels, which can offer relevant and sufficient information for the widest range of analyses typical in the literature. For example, when comparing the means of two interval variables, the most frequently reported results are the distribution of the two groups, the mean and standard deviation of the groups, the standardized effect size, the confidence interval (CI) of the group difference and the relevant hypothesis tests (chosen depending on the assumptions). These typical steps can be implemented relatively easily in an automatic analysis pipeline. (See a similar approach for fMRI preprocessing in Esteban et al., 2019.)

One critical question when designing such an automatic analysis pipeline is what analysis steps should be included? For example, which specific hypothesis tests should be used, how should the assumptions be checked, and what charts should be generated? Three straightforward sources can be readily identified. (1) In its simplest form, there are analysis methods and data processing steps that are mentioned in almost all introductory textbooks and which are required by the majority of scientific journals. In most cases, reporting some of the hypothesis tests is expected, reporting the effect size is highly recommended, checking raw data is necessary, and so on. As a default designing step, these points should be included in the analyses. (2) The methodological and 
statistical literature includes many improvements for standard introductory textbooks. There are many possible reasons why they are not included in mainstream textbooks: Some ideas are new, and textbooks usually include more established ideas; some ideas do not relate to mainstream topics and so are not included in textbooks, even if they could considerably improve the quality of reported results; moreover, some ideas have simply gone unnoticed. For example, median absolute deviation is a more appropriate outlier filtering method than the z-score-based solution (Leys et al., 2013), and improved single case methods offer more precise hypothesis tests than the previously used solutions (Crawford \& Howell, 1998); nonetheless, these methods are not as widespread as would be expected based on their value. If there is considerable agreement on some analysis methods, it is worth including them in the automatic analysis pipeline. If justified, these recommendations could replace textbook recommendations. (3) In other cases, there are various analysis solutions for a task, and several of them seem to work equally well. When a best solution does not exist, it is good practice to use a single solution consistently to make results more coherent and to make studies more comparable. For example, the $5 \%$ type I error rate is not chosen because $5 \%$ is much better than $4.9 \%$, but it was an arbitrary choice made within a reasonable range of values. Nonetheless, sticking to the $5 \%$ value is important because it makes studies comparable. Similarly, if there is no clear advantage to one procedure over another, selecting one of them and using it consistently in the literature makes studies comparable. For example, as mentioned in the previous section, there are many hypothesis tests for checking normality; among the relatively sensitive and powerful versions, it is not straightforward which one is the best. In such cases, any one of them could be chosen, but the solution should be applied consistently. See a similar example on arbitrary outlier exclusion criteria in Simmons et al. (2011). Overall, it is beneficial to use a single solution and to use it consistently across studies because it makes the studies more comparable.

Importantly, not all convincing and consensual recommendations found in the literature are suitable in themselves for automatic analysis. It is important to make a distinction between methodological works that make a recommendation to be included in an algorithm, and works that make a recommendation that depends on other unspecified considerations that are currently difficult to build into an algorithm. For example, if a methodological work suggests that a specific test is reliable only when a relatively large sample is used and also that the test should not be used with a sample smaller than 30, then this proposal can be followed clearly, and an automatic analysis can utilize it based on the sample size. On the other hand, if it is simply suggested that a small sample size makes the test less reliable, without specifying a threshold value, then this advice is hard to incorporate into an automated test, even if the proposal describes an essential feature of the test. For experts working on methodological issues, all kind of considerations are essential. However, in an automatic analysis, only the recommendations that can be added to an algorithm are applicable.

While it is essential to build upon consensual methods in an automatic pipeline, in the methodological and statistical literature, many methods are debated. One could go as far to say that it is hard to find any method that is not debated. Moreover, the consensual or debated status of an issue is also subjective. Due to the subjective evaluation of consensus and debates in general, it is 
important that the automatic data analysis pipeline design should be transparent and welldocumented so that the pipeline can be corrected and improved where necessary.

Such automatic analysis can improve the quality of reported results for a number of reasons. (1) Automatic data analysis software is useful because the analysis pipeline is fixed and standardized, meaning that there is a significantly lower possibility of p-hacking and, generally, it decreases the degrees of freedom for researchers (Bowring et al., 2019; Simmons et al., 2011; Wicherts et al., 2016). Overall, studies are more comparable when the decision criteria are standardized (Esteban et al., 2019). (2) The results can be more precise because, based on the automatic test selection and automatic result display mechanisms, it is less likely that the researcher will skip some relevant or even critical analysis steps or use some procedures incorrectly (Hoekstra et al., 2012). (3) Automatic analysis makes some procedures more accessible: Some lesser known and/or more appropriate and/or newer versions of a test or analysis can be used even if the researcher is less familiar with the analysis. Best practices of data analysis can be disseminated faster. (4) Because the analyses automatically display several related aspects of the data, it is less likely that the researcher analyzes the data "blindly", e..g., without checking some trivial preconditions, (such as whether outliers are seriously compromising the meaning of the results, or whether some distributions are bimodal) (Anscombe, 1973; Weissgerber et al., 2015; Wilkinson, 1999). (5) Because the analysis is simple in the sense that significantly less user interaction is required compared to traditional analysis software, the researcher can check more relevant details. (6) For statisticians and methodologists, automatic analysis software can be considered as a platform for creating recommendations and suggesting best practices. Automatic decision-based solutions can also highlight situations where the procedure should be changed or refined, which can facilitate methodological research and recommendations. It may also be able to facilitate recommendation types that can be described as an algorithm (in contrast to recommendations that cannot be described as a clear-cut decision tree, e.g., analysis of variance (ANOVA) is robust to normality assumption to some degree, where "some degree" is not clearly defined).

Importantly, automatic analysis does not exclude the possibility of individual analysis methods. When consensual standardized pipelines can be used, automatic analysis can be more efficient than individual analysis methods. However, when specific considerations require a unique selection of analysis steps, individual analysis steps should be chosen. Overall, automatic analysis can be a first (and often also the last) step in the analysis work, then, if needed, additional individual analyses can extend the automatic results.

These benefits of automatic analysis impact for many aspects of scientific works and statistics education. Researchers can perform analyses radically faster and more precisely, avoiding discouraged methods. This leads to improved report quality and helps to decrease the replication crisis. In addition, automatic solutions can be useful in education because the software can partly be considered as a tutor. 


\subsection{Arguments against automatic analysis}

While automatic data analysis may have advantages, as described above, it may also have its drawbacks. In this section, some common objections are considered and discussed.

Erroneous automatic pipeline. One may raise that if the automatic analysis steps are incorrect, then the computed results will also be incorrect. While this is a valid issue, in fact, it is not an automatic analysis-specific problem. Because the analysis steps are based on the statistical and methodological literature, potentially incorrect analyses only reflect the incorrect choices in said literature. In other words, if some recommendations from the literature are incorrect, then the automatic and non-automatic solutions that follow the recommendations will both be incorrect. Therefore, the root of the problem is not in the automatic nature of the software but rather in the incorrect recommendations from the literature, and it is the literature where the issue should be fixed.

Debated recommendations. Many methods are debated, and one may worry that, if these methods are included in an automatic analysis pipeline, researchers will use them. This issue is similar to the erroneous procedures issue, as discussed above, because debated recommendations are potentially erroneous methods. In line with this similarity, as in the case of the erroneous pipeline, this concern may be true; however, it is also not specific to automatic analysis. Debated methods can also be used by researchers in non-automatic analyses, and it is the methodological and statistical literature that should resolve these debates. Nonetheless, there are potential measures that automatic analysis software could take. When a method is strongly debated (even if contentiousness is somewhat subjective, as discussed above), developers may decide that these methods should not be included in the pipeline until the debate is resolved. Alternatively, debated methods could be labeled in the output or could be turned on and off in the preferences.

Rigid automatic pipeline. It may be raised that if the processing pipeline is fixed, then researchers cannot perform any analysis that requires any modification of the standard procedure. This would block any creative and innovative solution or any appropriate solution that is beyond the rigid pipeline. In fact, using modified solutions is not antithetical to automatic and standardized analysis methods. As in other cases, a researcher can at any time decide to use additional or modified analysis methods. This is similar to the procedure for a pre-registered study, wherein additional analyses are introduced after learning the results of the planned analyses. The critical condition is that, in these cases, the researcher should give appropriate justification for the alternative procedure. Automatic analysis can make this requirement more transparent and explicit: By default, researchers should use the recommended standardized methods and apply alternative solutions only if appropriate justifications are given. Currently, this is often not the case. For example, the use of ANOVA without a normality assumption check is frequently justified by the observation that ANOVA is somewhat robust to the violation of the normality assumption. One significant problem with this justification is that the meaning of "somewhat" is unclear: How strong exactly should the normality violation be to use an alternative hypothesis test? Unless an acceptable level of robustness is defined, reference to the robustness of the ANOVA rule is arbitrary. Fixed pipelines, on the other 
hand, set more straightforward reference points that help to define what decisions should be justified.

Similar results across methods. One may also raise that one should not worry too much about the slight differences between the automatic analysis pipeline and some similar non-automatic solutions, because most methods provide the same result (e.g., most hypothesis tests provide a significant result if the effect is strong enough). Therefore, the advantage of the optimized automatic analysis pipeline is inessential. Note that, unlike previous concerns that worry about the use of the most appropriate method, this concern argues that the importance of slight differences is overestimated. However, a large part of the replication crisis literature demonstrates that these slight differences and biases add up to create a massive bias in the publications, leading to a crisis in many research areas and to doubts about the scientific approach (Carp, 2012a; Open Science Collaboration, 2015; Silberzahn et al., 2018; Wicherts, 2017). Therefore, improved methodology, better research practices, and reasonable recommendations are essential in advancing the scientific method and practice.

A black box cannot advance statistical understanding. Another concern may be that, by using an automatic data analysis method, statistical procedures will become black boxes for users, and, therefore, researchers will not understand how the results were found and what they mean. Relatedly, instead of automatic analysis methods, statistical procedures should be taught appropriately, so researchers can apply the analysis methods appropriately.

However, the proposed software type is not necessarily a black box. If the output reveals essential properties of the data, automatic analysis can be more informative than the usual practice that researchers follow; more informative and more accessible output helps in the understanding of statistics. Appropriate output turns the procedure into a glass box, instead of a black box. Moreover, carefully designed output will advance statistical understanding. The following sections discuss how careful compilation of the results prevents the results from becoming a black box.

Need for additional software packages. If the analysis pipeline is fixed, then any alternative analyses require additional software packages. Theoretically, it is not necessary for automatic and individual analyses to be performed in different software packages. However, currently only CogStat implements automatic analyses and all other packages provide individual analyses (though see examples of partial automatic analyses below.) Nonetheless, this issue is also not limited to automatic solutions. All software solutions have their limitations: What analyses are available, how easy and fast it is to launch the analyses, how easy it is to read and understand the results, etc. Usually, researchers use their preferred software package with which they can solve most of their analysis tasks. When the preferred software reaches its limit, researchers routinely use other software packages to perform an analysis that their default software cannot solve (e.g., GPower for power analysis and JASP for Bayesian hypothesis tests). Overall, automatic analyses require the same procedures that researchers apply with non-automatic analysis software, even if many researchers can imagine a better feature combination of their favorite software packages. 


\section{Need for optimized result reports}

In an automatic data analysis software, it is even more critical what is displayed in the results. The present section summarizes some key considerations that can make the output more understandable, more accessible, and more concise.

It is essential what information is reported about empirical data and what information is offered in analysis software solutions. (a) For example, it has been demonstrated that the p value of hypothesis tests is often misunderstood (Nickerson, 2000), and several alternative indexes have been proposed as alternatives for the $\mathrm{p}$ value or, more generally, as alternatives for frequentist hypothesis tests (Cumming \& Finch, 2005; Killeen, 2005; Nickerson, 2000; Wagenmakers et al., 2017). Thus, the information provided in reports has an effect on whether analysis results will be interpreted and handled correctly. (b) As a negative example, several analysis software solutions provide intermediate calculations that are usually not reported or not interpreted in themselves, and these intermediate results may cause confusion for some users (see the example of the sum of squares in ANOVAs below). (c) As evidence of the importance of the software output, the structure of the output may be considerably different than the required format in the reports. For example, the table format of degrees of freedom in ANOVA is substantially different in many software solutions from the format required in APA specification. In light of the fact that a considerable number of erroneous reports are rooted in copy-paste errors (Bakker \& Wicherts, 2011), this is an issue that should be handled.

Overall, it is essential what numerical results and visualizations are reported and what results are provided by an analysis software. This is especially important in an automatic analysis pipeline where all components of the output are compiled entirely by the software. A few general viewpoints on designing more informative output are listed here, while more specific solutions are provided in the next section, which is where CogStat-specific solutions are presented.

Displaying only the relevant results. Many software solutions provide details that are irrelevant for most users, which should be avoided in an optimized report. To follow this principle, it is essential to define what results are relevant. Some of the results that many statistical software display are directly usable for the researcher in the sense that the results give critical information about the investigated data. For example, standardized effect sizes, confidence intervals or the $p$ values of the hypothesis tests give essential information about the given sample and about the population from which the sample originated. Some other results in themselves are less useful because they are intermediate results for calculating the relevant results. For example, the sum of squares values of ANOVA are useful to calculate the test statistics or the effect sizes, but they are less useful in themselves; in other words they are useful only in the sense that they provide indirect information about relevant indexes. There could be indexes wherein it is less trivial to decide how relevant they are. For example, the degrees of freedom in hypothesis tests could be ambiguous because there is no direct need to know the degrees of freedom to calculate the p value (i.e., the computer will handle this); nonetheless, it is relevant in the sense that APA format reporting requires this information. As another example, some may believe that this information is relevant to 
check whether the analyses were performed correctly. However, degrees of freedom and similar indexes are, in fact, arbitrary in analysis checks: Why not ask for other details of the calculation? In this case, checking is possible because the degrees of freedom information is redundant, which enables the monitoring of the analysis, and the fact that this information is redundant shows that it is an unnecessary piece of information. To summarize, the results that can be used directly to understand and interpret the data should be used in the output.

Displaying the data in graphical format. Graphical depictions of data are often easier to grasp compared to equivalent numerical presentations (Wilkinson, 1999). At the time when computers started to be a common part of research practice, Anscombe (1973) noted that "a computer should make both calculations and graphs. Both sorts of output should be studied; each will contribute to understanding." Even almost 50 years later, when computers and capable software solutions are an essential part of the research practice, this advice still needs to be warned about (Weissgerber et al., 2015). Although presenting the data both numerically and graphically may sound redundant, it is not because graphical presentation provides faster grasp of the critical information, while numerical presentation provides more precise information.

Avoiding blind analyses. Focusing only on a few pieces of information, instead of a richer set of details, can lead to an incorrect or biased interpretation of the results. For example, key descriptives in themselves do not straightforwardly show various patterns in the raw data (Anscombe, 1973; Weissgerber et al., 2015; Wilkinson, 1999). As another example, focusing only on the hypothesis test result can result in missing critical information about statistical power or the precision of the analysis (Cumming, 2014; Cumming \& Finch, 2005; Faul et al., 2007; Nickerson, 2000). Overall, focusing only on a few key properties can lead to a blind analysis. However, in an automatic analysis software, it is simple to provide a larger set of complementary properties of the data, so that the user can quickly review all these relevant details to obtain a comprehensive view of the data.

Displaying graphically the information on which the analyses rely. When charts are shown, it is important that they display the information that illustrates the key properties of the data. While this principle sounds trivial, there are several counter examples of this consideration not being used widely. (a) For example, analyses working on ordinal variables utilize or "see" only the rank information of that variable and not the original values, and Spearman correlation is a Pearson correlation on the rank information. Accordingly, for ordinal variables it is more appropriate to display the rank information, instead of the original values. (b) As another example, when using repeated measures variables, it is a critical piece of information that the data are in pairs, and this information is utilized in the analysis. Furthermore, when comparing the means of two interval variables, it is the difference within the cases that is tested. However, this information is not understood by many practitioners, and this information is usually not displayed either (Weissgerber et al., 2015). This principle is strongly related to other formerly mentioned principles as well: Providing information in charts that is relevant in the analysis is in line with giving relevant information, including graphical presentation, and avoiding blind analysis. 
Formatting the results in the style of the report. Frequently, in analysis software, the results are not displayed in a format that can be transferred directly into a report. For example, a hypothesis test's degrees of freedom is displayed in a column of a table, while the APA format requires them to be shown in parentheses before the test value. For the researcher, it is easier to handle the output if no additional transformation is required, and the output can be directly copied to the manuscript; otherwise, there is the risk of transformation errors, leading to erroneous reports (Bakker \& Wicherts, 2011). If different styles are required in different research areas, then software preferences should be used to handle different reporting styles—similar to the reference style settings in citation management software.

\section{An implementation of an automatic data analysis software: CogStat}

Following the considerations discussed above concerning automatic data analyses and optimized output, the present section introduces an automatic data analysis software. CogStat includes solutions that rely on the considerations discussed above and also includes additional innovations to make analyses faster, more understandable, and more coherent. The present section introduces the main distinguishing features of CogStat. After a short description of some general features, the first subsection presents how CogStat can technically be used, highlighting its differences from traditional statistical software. The second subsection presents how the viewpoints of automatic analyses and optimized output are implemented and what additional specific innovations are needed to make the software more usable. The last subsection presents how the described analysis method can be efficient.

As a possible implementation of the proposal above, CogStat, an open source and cross-platform statistical package, was developed which is freely available at https://www.cogstat.org.

In CogStat, after the user specifies the task, the variables, and the measurement levels of the variables, the software selects the relevant analysis steps, displaying the most relevant results in numerical and graphical format, and the relevant results are displayed in a format that is used in reports, e.g., the American Psychological Association (APA) style. As of version 2.1, CogStat supports several functions related to exploring a single variable, exploring a variable pair, comparing repeated measure variables, comparing groups, and calculating pivot tables. In most analyses, raw data are displayed graphically, relevant descriptive statistics (including standardized effect sizes) are displayed numerically as well as graphically, and inferential statistics are shown including point and interval parameter estimations (also the standardized effect sizes), hypothesis test with sensitivity power analysis and with appropriate assumption tests. For a more specific description of what analyses can be conducted in CogStat, see the online documentation of the software.

CogStat was initially made primarily with cognitive psychology research in mind, meaning that some of the most frequent tasks in that field are implemented first, and it supposes relatively small samples. Nonetheless, many other research areas wherein the same data analysis or statistical 
solutions can be applied (e.g., biological research, linguistics, cognitive neuroscience, psychology, and educational research) can use CogStat.

CogStat is localized to many languages: As of version 2.1, it is available in 19 languages. CogStat is a cross-platform software, supporting Windows, macOS, and Linux systems. CogStat can be run not only in its own graphical user interface, but also in Jupyter notebook. In a Jupyter notebook, CogStat commands are available as Python methods and can be used with any Python script that analyzes other aspects of the data.

Calculations in CogStat are validated by comparing its calculations with the results of other statistical packages (including SPSS, R, and jamovi). In some special cases, because of methodological or technical considerations, CogStat and the validator software solutions use different versions of some methods. See a list of those methods in the online documentation of CogStat.

The software is written in Python, which is a high-level general language popular among researchers. CogStat usually relies on other Python modules to run specific analyses: Data tables are handled by the pandas module (McKinney, 2010), most statistical calculations are supported by the scipy stats (Oliphant, 2007), the statsmodels (Seabold \& Perktold, 2010), and the pingouin Python modules (Vallat, 2018), and figures are created with the matplotlib module (Hunter, 2007). Therefore, CogStat technically can be considered (a) as a more convenient GUI for specific analyses that displays the results in specific ways, and (b) as a compilation mechanism that selects the specific analyses based on the question and the measurement level of the variables. (See more details on the technical background in the online developer documentation.)

\subsection{Practical use of CogStat}

Technically, CogStat is simpler to use compared to traditional statistical software. After installing CogStat, data analysis includes only a few steps.

Step 1: Data import. CogStat does not include a data editor, so it is recommended to store and edit the data in spreadsheet editors, such as Microsoft Excel or LibreOffice Calc (Figure 1A) (see a similar solution in JASP: Wagenmakers et al., 2017) or in other statistical software formats that can be imported to CogStat, such as SPSS or jamovi. Beyond the variable names and the data themselves, it is important to include the measurement levels in those data files because automatic data analysis heavily relies on that information. In some statistical software package formats, such as SPSS, the measurement level property should be set in the appropriate user interface, while the measurement level should be added as the second line in a spreadsheet software (see Figure 1A for an example dataset).

Step 2: Analysis. After the data are imported to CogStat, an analysis task can be chosen from the Analysis menu, such as “explore variable”, and “compare groups” (see Figure 1B for all available analysis tasks and see more details on the analyses in the online documentation). Note that, unlike in traditional statistical software, the menu does not include specific hypothesis tests, graph types, or data analysis steps, but it includes the analysis tasks that the researcher wants to solve. In the 
analysis task dialog, the variables and some options should be specified (Figure 1B), and then the analysis can be run.

Step 3: Results. The results will be displayed in the output area (Figure 1C), from which they can be copied to the clipboard (and inserted into any other software that supports clipboard, i.e., the copy-and-paste method) or saved as a pdf file.

Step 4 (optional). Additional individual analysis. If the results of the automatic analyses show that additional specific analysis steps are required, supplementary analyses can be performed in the traditional way. Because CogStat does not support individual analysis at this time, traditional statistical analysis software packages can be used instead. 
A

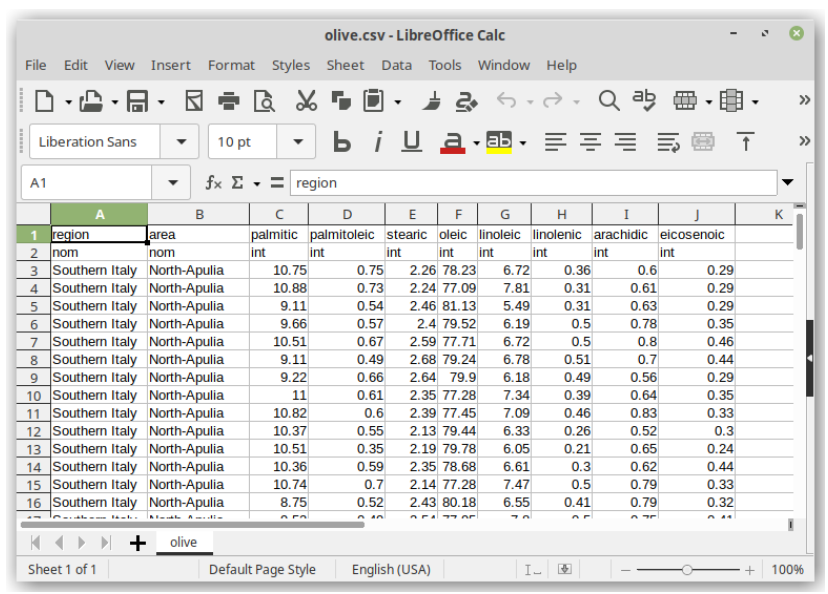

$\mathrm{B}$
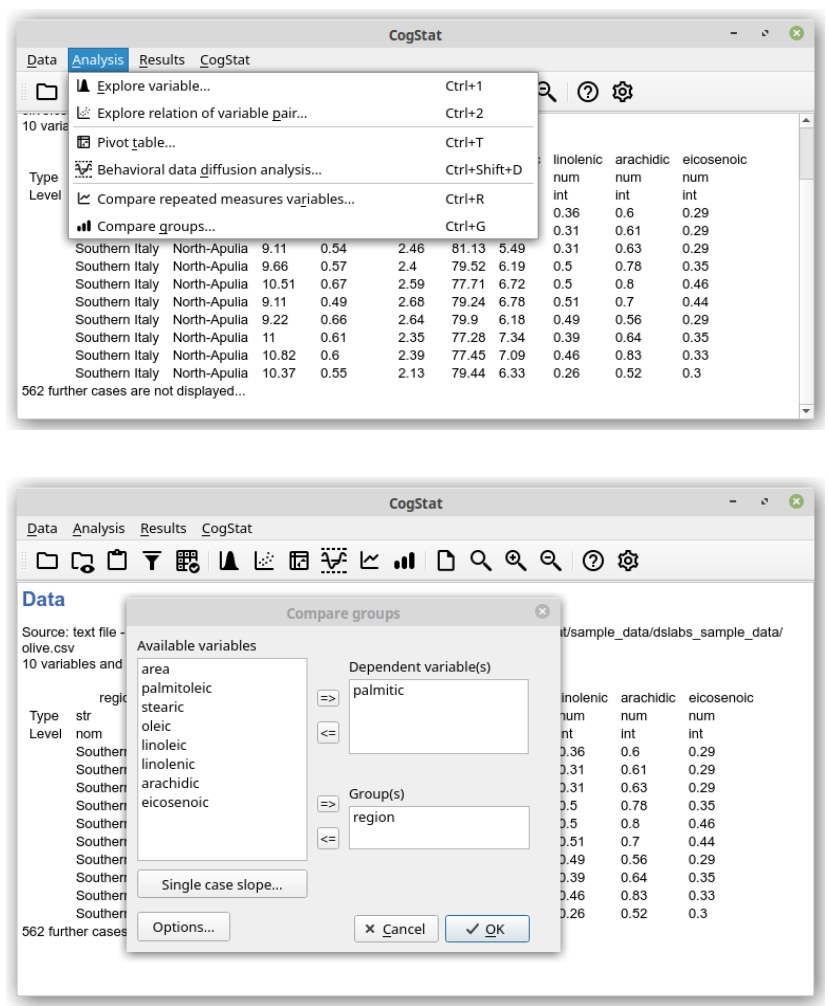

C

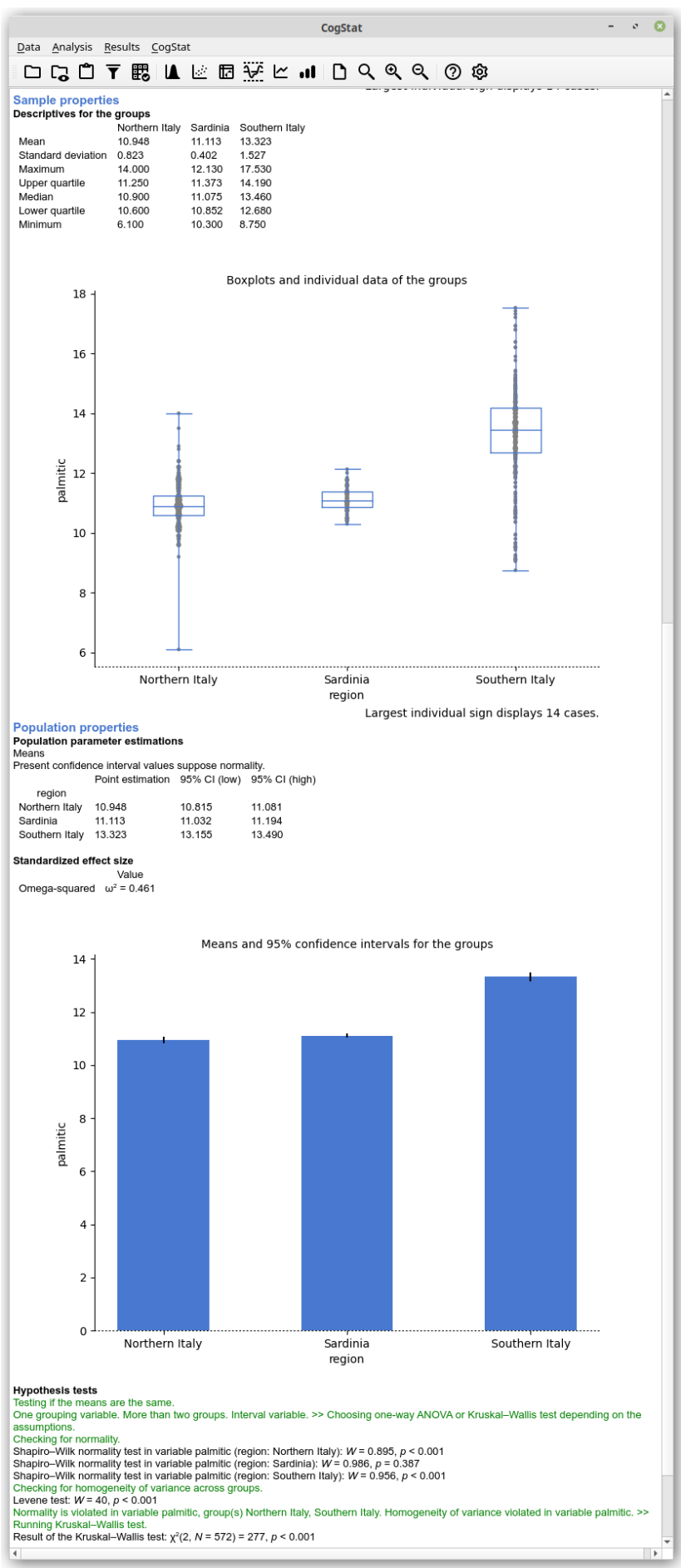

Figure 1. A Example dataset stored in LibreOffice Calc spreadsheet software. First row includes the variable names, second row stores the measurement level, and remaining rows include the values. B Top: Selecting an analysis task in CogStat. Bottom: Setting variables for group comparison task. C Results of group comparison task. 
See more details on the practical use of CogStat in a Quick Start guide and other more detailed descriptions in the online documentation of the software by choosing the CogStat > Help menu in CogStat.

\subsection{Designing the analysis pipeline in CogStat}

In line with general considerations concerning automatic analysis as proposed above, CogStat implements analysis pipelines that include several additional innovations specific to this software. In the case of some specific features, similar features are available in other software solutions (such as the pandas-profiling Python module for automatic analyses, see Brugman, 2016/2021), but CogStat provides the most comprehensive set of features to implement the automatic analysis method.

Before describing some of those specific properties in CogStat, we briefly review the main considerations by which these pipelines are designed. In CogStat, there are four sources of the pipeline design decision. The first three are the ones that were mentioned above in the The possibility of automatic data analysis section: (a) following the most common textbook practices, (b) following the additional recommendations of the methodological and statistical literature, and (c) following that, when there are equally appropriate solutions, one of the most commonly used solutions is chosen. There is a CogStat-specific fourth source: (d) CogStat includes unique innovations for how the results should be structured or displayed (see a few examples below in the present subsection). Occasionally, technical implementation can cause interim constraints: Because some of the preferred solutions may not be available in Python modules, the best solution that is available in Python is used in the CogStat analysis pipeline. The details of the specific pipelines and decision trees and their justifications can be found in the online user documentation, in the online developer documentation, and in the online issue tracker. In addition, the issue tracker and discussion forum are available for anyone to share ideas and suggestions. Therefore, the chosen analysis pipeline is transparent and can be discussed openly.

When designing the analysis pipeline, there are general considerations or principles, and there are more specific ideas that extend and specify the general principles. First, we review some of the general principles of the CogStat analysis pipeline design, and then some specific design points are listed.

Display only the relevant results. As described above (in section Need for optimized result reports), results are considered relevant if they directly show some essential property of the data (e.g., results of the hypothesis test, interval estimation, standardized effect sizes, and main descriptives), and results are less relevant if they are intermediate calculations for relevant results. In CogStat, most analyses display the same type of information in the same order (e.g., raw data in graphical format, main descriptives, standardized effect sizes of the sample, and interval estimations), and only the results providing these properties are displayed, ensuring that only the relevant results are displayed. 
Display the data in graphical formats. In line with the principle that was discussed in more detail above, CogStat frequently presents the results in graphical form. The same information is often shown in both graphical and numerical forms: Graphical forms are for grasping the broader aspects of the data, while numerical results can be used for a more precise report.

Data-driven analysis. This principle means that the analysis should primarily depend on the data (and, obviously, on the task), not on the potentially subjective or arbitrary decisions of users. This principle ensures that the analysis is objective in the sense that the same data will produce the same results independent of the user. Data-driven analysis decreases unnecessary degrees of freedom for researcher (Bowring et al., 2019; Carp, 2012a; Simmons et al., 2011). For example, there are proposals to ensure the normality assumption by visual inspection (Schucany \& Tony Ng, 2006; Wilkinson, 1999); this would require additional subjective user-based decisions. Instead of visual inspection, the hypothesis test of normality does not require additional user-based decisions. Note that, based on the graphical depiction of the data, the researcher can still decide that the hypothesis test did not capture a violation of the assumption and use another non-automatic software for a more appropriate analysis. However, this should not be a recurrent issue. Because of data-driven analysis, CogStat assumes that the data has already been collected; therefore, no pieces of advice are given as would be in a planning stage. For example, in CogStat, no a priori power analyses are given to calculate the necessary sample size for a study; instead, the sensitivity power analysis (i.e., the minimum effect size for the given sample size that provides appropriate power) is calculated to help evaluate the hypothesis test result. Note that sensitivity power analysis is not equivalent to post hoc power analysis where power is computed and which can be used inappropriately when one considers the calculated sample effect size as a population parameter (Faul et al., 2007).

APA format. CogStat was initially made with cognitive psychology in mind; because most journals in cognitive psychology require the results to be reported in the APA format, when it is relevant, CogStat displays the results in that format (see a similar approach in JASP: Wagenmakers et al., 2017). This feature makes report writing more efficient and can make writing more error-proof (e.g., some of the errors in reports are simply caused by an erroneous copy-paste method: Bakker \& Wicherts, 2011).

Display raw data. While summary statistics reduce redundancy in the data, there can still be unexpected details within the raw data that cannot be captured purely by the summary statistics. For example, fundamentally different data can lead to the same numerical descriptive results; summary statistics cannot reveal outliers, unexpected deviation from the common normal distribution, or other unexpected patterns (Anscombe, 1973; Matejka \& Fitzmaurice, 2017; Weissgerber et al., 2015; Wilkinson, 1999). Therefore, many textbooks suggest that raw data should be checked in the first data analysis steps. Relatedly, in several cases, it is recommended to report the raw data, so that readers can form their own evaluation of the data. However, researchers may skip the raw datacheck step partly relying on their former experience with similar data wherein no unexpected patterns were observed (Anscombe, 1973; Wilkinson, 1999); in doing so, they save time by not performing an analysis step, which usually does not provide any surprising details. In reports, while 
scatter plots that include individual data are reported regularly, other analyses, such as group comparison, often do not present raw data (Weissgerber et al., 2015).

CogStat offers various solutions to ease raw data evaluation. First, in most analyses, the first section of the output displays the raw data without any additional information (see an example in Figure 2A). This enables researchers to evaluate the data without any summary or other result that could influence or bias their evaluation. With this information, researchers can investigate whether there are unexpected patterns in the data that usual summary statistics cannot capture, what results can be expected for the main questions (e.g., can a difference between two groups be expected?), and whether additional actions are needed to process the data. Second, in most analyses in CogStat, summary statistics (e.g., the box plot of the distribution) are displayed in a way that the raw data are visible in the background. This gives more background information on how the summary statistics are formed. Displaying raw data separately and together with some descriptives leads to some redundancy, but it is justifiable to repeat the same information in different contexts because these charts serve different purposes and can be used differently. Overall, CogStat displays the raw data because it helps researchers and students to interpret their data more appropriately (Anscombe, 1973; Weissgerber et al., 2015), while keeping the analysis simple in terms of user interaction.

Strict separation of sample and population properties. In almost all empirical studies, only a portion of the whole population is measured. It is very rare that the data of the whole population can be collected. When a sample is measured, descriptive statistics provide information about the sample, and inferential statistics provide information about the population. It is essential to keep this distinction in mind while interpreting the results, because, while sample properties are hard facts, and those are the properties of the collected cases, population properties are always uncertain to some degree, which is rooted in the unavoidable sampling noise; therefore, inferential results are always partly speculative.

To highlight the difference in the interpretation of descriptive and inferential statistics, CogStat displays sample and population properties strictly separated (Figure 1C). In the rare cases when the whole population is measured, the sample properties section of the output can be considered as the description of the population, and the population properties section should be ignored. In addition, separate sample and population property information provide insight on how the inferential statistics are formed, in other words, what raw data the inferential statistics rely on. See an example of how descriptive and inferential data when displayed side by side can provide complementary and more comprehensive information about the data in Krajcsi (2021).

In various cases, the strict separation of the sample and population properties can lead to seeming redundancies. For example, the descriptive mean of an interval variable is the same as its population point estimation. Nonetheless, it is important to highlight that, as a sample property, the mean reflects the hard fact about the cases that have been measured, while, as a point estimation, it is only a best guess.

Display graphically the information that is relevant in the analysis. Research and reporting practice often fail to present the relevant information on which an analysis relies. CogStat highlights 
that information on several occasions. (1) Put ordinal information on charts. In ordinal variables, the relevant information on which the statistical analyses rely is the rank of the cases. In line with this viewpoint, CogStat charts showing ordinal variables display the rank of the values (Figure 2B) (axes are displayed with a dashed line; see more details below). (2) Change between conditions in repeated measures data. As mentioned in the previous section (Need for optimized result reports), in repeated measures data, analyses utilize the fact that some of the data come from a single participant. In CogStat, when repeated-measures interval variables are compared, the individual data of the same cases are connected, highlighting the status of the repeated measures variable and displaying the important aspects of the data on which the analysis relies (Figure 2C). (3) Highlight measurement levels on charts. To highlight the measurement levels on charts and to simplify the interpretation of the analysis, CogStat displays the axes in line with the measurement level of that variable. Interval variables are displayed with solid lines, ordinal variables are displayed with dashed lines to reflect the unknown distance between neighboring values in the scale, and nominal variables are displayed with dotted axes to reflect the lack of order in that scale (Figure 2). In line with the principle that information relevant to the analysis should be shown, in some analyses, the original measurement levels of the variables are overridden. For example, in group comparison analyses, grouping variables are handled as nominal variables even if they include higher measurement level information, such as education level, which is at least an ordinal variable. In these cases, grouping variable is displayed as a nominal variable (Figure 2A and 2B). 
A

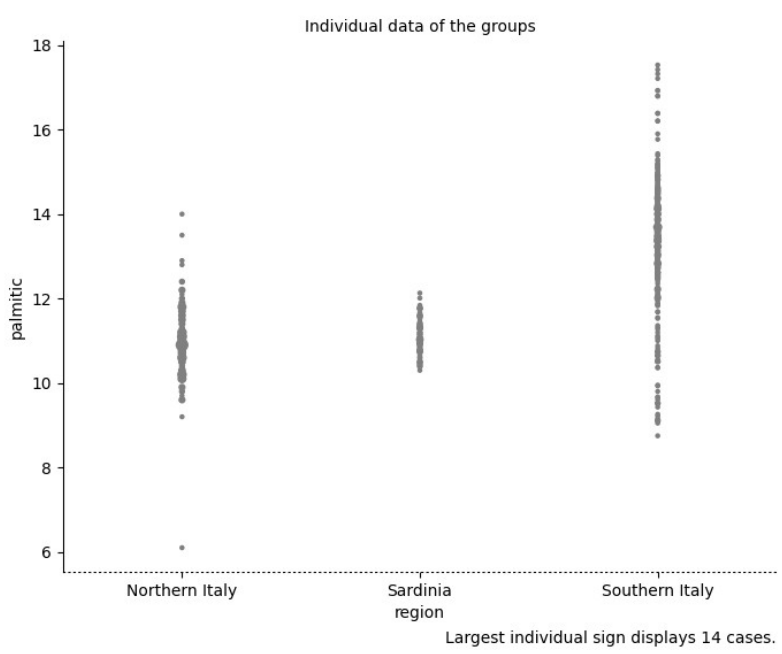

B

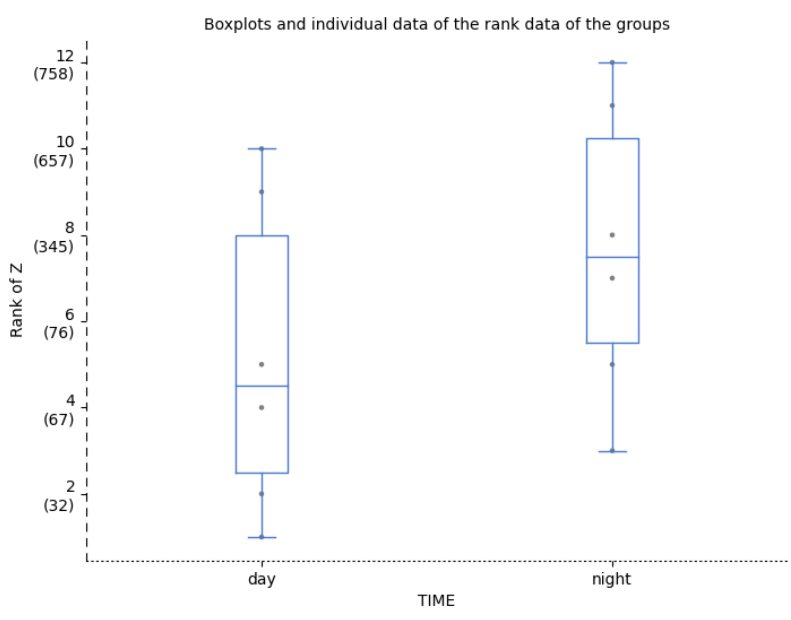

C

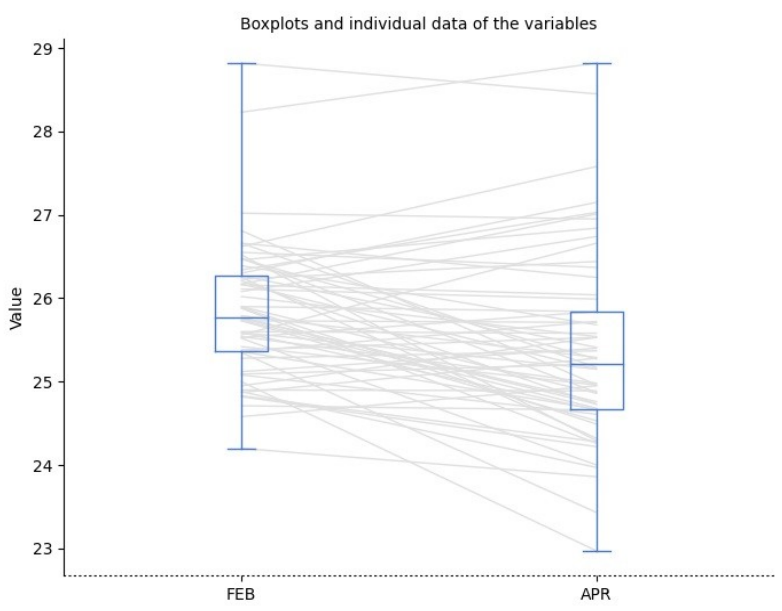

Figure 2. Example charts. A Raw data of three groups. B Comparison of two groups of ordinal variables. C Comparison of two interval repeated measures variables.

Standardized effect sizes. Unlike the frequently used p values of hypothesis tests, effect sizes provide a description of the observed effect size without the influence of the sample size (Fritz et al., 2012; Wilkinson, 1999). Effect sizes help to compare different studies and to evaluate the practical significance of the finding. The confidence interval of the effect size indicates the precision of the estimation; however, effect size CIs are rarely reported (Fritz et al., 2012).

CogStat calculates the effect sizes in several analysis, displaying them in a separate subsection of the results, while also separating the sample effect sizes and their population estimations. In several cases, the confidence intervals of the population estimations are also provided (see also the following point).

Confidence intervals. It has been proposed that, instead of using hypothesis tests or using them exclusively, interval estimations should be applied because they offer more direct and quantitative values for relevant parameters (Cumming, 2014; Cumming \& Finch, 2005; but see Morey et al., 
2016). These direct estimations are easier to interpret than the results of hypothesis tests (Coulson et al., 2010; but see e.g., Hoekstra et al., 2014). In addition, interval estimations provide an estimation of the precision of the measurement (Cumming \& Finch, 2005).

CogStat provides interval estimations in several analysis in the form of confidence intervals (CI). Importantly, to highlight the informativeness of CI over hypothesis tests, CIs are displayed before the hypothesis test section (Figure 1C).

Automatic hypothesis tests. One of the most straightforward parts of the automatic analysis pipeline design is the selection of the automatic hypothesis test: Most textbooks agree upon how the appropriate tests should be chosen, and, for the most frequently used tests, these agreements can be easily implemented in an automatic pipeline. Overall, based on (a) the aim of the analysis, (b) the measurement level, and (c) the assumptions, it is straightforward which hypothesis tests should be used. In CogStat, hypothesis test selection is entirely automatic, and the user cannot change the selected tests. When selecting the hypothesis test, CogStat displays information on what and why it was selected (see an example in Figure 3). There are similar solutions in other software packages, that use a wizard-like user interface solution to aid the selection of the appropriate test (e.g., SOFA, the Statkat module of jamovi, and InStat: GraphPad Software, n.d.; Paton-Simpson, n.d.; The jamovi project, 2020), or the test selection is partly automatized (as in mbir: Peterson \& Caldwell, 2019; or in ROPstat: Vargha et al., 2015), but even so the CogStat implementation is intended to be more comprehensive and entirely automatic. (See the decision tree of test selection in CogStat in the online documentation, as listed in the Analysis menu explanation sections.)

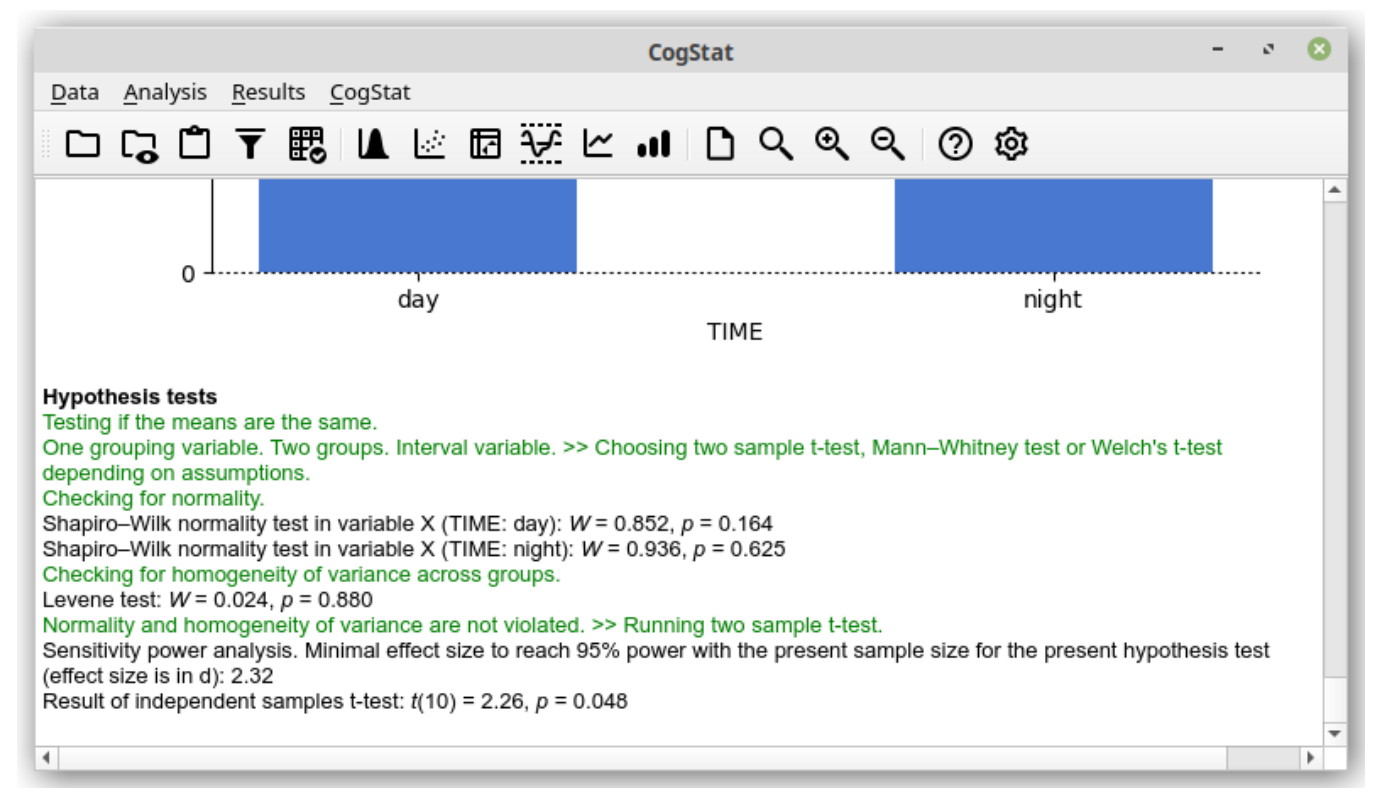

Figure 3. Example of automatic hypothesis test selection.

Sensitivity power analysis. Power analysis is essential to understanding and planning the use of hypothesis tests (Button et al., 2013). Power analysis connects type I errors, type II errors, effect sizes, and sample sizes: If three of these parameters are known, the fourth can be calculated (Faul et al., 2007). Among the various power analyses, sensitivity power analysis is in line with the data- 
driven analysis philosophy of CogStat. Sensitivity power analysis can specify the minimum effect size that can provide the appropriate power with the given sample size (Faul et al., 2007). In CogStat, sensitivity power analysis is run after the software chooses the appropriate hypothesis test (e.g., after running the appropriate assumption checks), and the result is displayed before the hypothesis test results (Figure 3). This solution highlights the fact that power analysis is related to the hypothesis test but not to other methods that investigate the population properties, such as the interval estimates. Automatic sensitivity power analysis in CogStat also highlights the role of power analysis in thinking about and interpreting hypothesis test results (Button et al., 2013).

\subsection{Efficient analysis in CogStat}

By using automatic analysis, CogStat is more efficient than many other software solutions.

(1) CogStat requires less user interaction to obtain the same results than other statistical software.

Table 1 illustrates the radical difference between the number of user interactions in CogStat and in a popular statistical software, SPSS. In CogStat, analyses require so few user interactions that one may consider not saving the results at all because saving and reopening the results versus importing the data and rerunning the analysis are comparable in terms of user interactions and time. (2) The output is optimized and structured for faster review and easier interpretation; it intends to provide a comprehensive picture of the research question. Optimized and structured output can help researchers to find the relevant information faster and can include relevant information for a more complete evaluation of the data. (3) Researchers using other statistical packages may spend more time finding rarely used analysis methods and also learning how to perform them on the software. In CogStat, all details are provided automatically, so there is no need to look for the appropriate user interaction. In addition, in CogStat, the analyses result output shows similar subsections in the same order, independent of the task, the measurement levels, or other conditions; therefore, even if the researcher does not know a specific analysis method (e.g., a specific hypothesis test), subsection titles and other explanatory messages of the output make it effortless to understand the function of the analysis. Obviously, understanding the bases of statistical analyses is necessary to understand the analysis report (e.g., if a researcher does not know what a hypothesis test, confidence interval, or sensitivity power analysis is, then the analysis output cannot be interpreted and used appropriately), which is the case for any statistical software output. 


\begin{tabular}{|c|c|}
\hline Required steps in SPSS (v25) & Required steps in CogStat (v2.1) \\
\hline $\begin{array}{l}\text { 1. Graphs }>\text { Graphboard template chooser } \\
\text { 2. Set dependent and grouping variable and select 2-D } \\
\text { Dot Plot } \\
\text { 3. Hit OK } \\
\text { 4. Analyze > Descriptive Statistics > Explore } \\
\text { 5. Set dependent and factor list } \\
\text { 6. In Plots, set Normality plots with tests, and hit } \\
\text { Continue } \\
\text { 7. Hit OK } \\
\text { 8. Find the normality test in the output, and check if } \\
\text { normality is violated } \\
\text { If normality is not violated: } \\
\text { 9. Analyze }>\text { Compare means }>\text { Independent-Samples T } \\
\text { Test } \\
\text { 10. Set the test and grouping variables } \\
\text { 11. In Grouping variable, set the group levels, and hit } \\
\text { Continue } \\
\text { 12. Hit OK } \\
\text { 13. Find the Levene's test, and depending on the result, } \\
\text { find the appropriate test result } \\
\text { If normality is violated: } \\
\text { 9. Analyze }>\text { Nonparametric tests }>\text { Independent samples } \\
\text { 10. Set objective to Compare medians across groups } \\
\text { 11. On Fields, set test fields and groups } \\
\text { 12. Hit Run } \\
\text { 13. The result misses test statistics (and only includes the } \\
\text { p value), that a reviewer may also miss } \\
\text { 14. SPSS does not calculate Cohen's d and Hedges' g, so } \\
\text { calculate it by hand or with custom SPSS syntax file } \\
\text { 15. For sensitivity analyses you need SPSS Statistics Base } \\
\text { Edition. In Analyze > Power Analysis > Means > } \\
\text { Independent-Samples T Test, set the variables, choose } \\
\text { Plot and enable Power estimation versus effect size. }\end{array}$ & $\begin{array}{l}\text { 1. Analysis > Compare } \\
\text { groups } \\
\text { 2. Set dependent and } \\
\text { grouping variables } \\
\text { 3. Hit OK }\end{array}$ \\
\hline
\end{tabular}

Table 1. Typical analysis (i.e., suggested by most textbooks) steps in SPSS and in CogStat for comparing the mean of two groups.

\section{How automatic analysis and optimized output can be beneficial?}

An automatic analysis software is potentially useful for various groups of users. In the present section, it is briefly summarized why different groups of users may find such a software package 
beneficial. It is also summarized how the replication crisis, statistics education and data analysis methods can be improved.

Researchers. Researchers may find automatic analysis software useful for several reasons. (1) Most importantly, automatic analysis is much faster compared to individual analysis methods, because significantly less user interaction is required in the analysis software (Table 1). (2) The results can be reviewed more quickly, because they are optimized to display the relevant aspects of the data. (3) The results are more precise because the automatic pipeline handles several details that are not handled by many researchers (Hoekstra et al., 2012) and because the data are presented in a format that is more similar to the publication format. (4) Analyses are more accessible because researchers can use a specific analysis method (e.g., a new hypothesis test, a new confidence interval calculation method, or a new standardized effect size) even if they do not know that particular method - supposing that they understand what the general category of the analyses (e.g., hypothesis test, confidence interval, or standardized effect size) can be used for (see similar arguments in Wagenmakers et al., 2017; Wallace et al., 2012). The first two advantages apply to all researchers, including scholars who are comfortable with most nuances of statistical analyses. The last two advantages will be useful for researchers who are unfamiliar with all the relevant considerations of statistical analyses.

Novice users. Automatic analysis software may also be useful for novice users who are learning statistics. (1) It is easier for them to find the appropriate analyses because the menus indicate the tasks, not the name of hypothesis tests or other statistical terms. (2) With more relevant details in the output, parallel numerical and graphical display of the results, optimized charts, and additional features of the optimized output, it is easier to find and interpret the relevant information. (3) In CogStat, initial use of the APA format is also assisted by the appropriately formatted output. Overall, automatic analysis software can be considered as a tutorial system for beginners.

Methodologists. In the methodological literature, it is a common debate what procedure is ideal for a specific task. For this reason, automatic data analysis software packages can be considered as platforms for accumulating best practices for data analysis. A software that runs analysis partly automatically can be seen as a field for creating consensus.

Mitigating the replication crisis. Automatic data analysis solutions with optimized result reports can help to overcome the replication crisis in several ways. In an automatic analysis software, the results are more precise, the researchers' unnecessary degrees of freedom are decreased, and the results are easier to understand and interpret. The automatic software could also serve as a platform to form consensus and to make advantageous methods more accessible. All of these features contribute to more precise and bias-free reported results, mitigating the replication crisis.

Efficient analysis. Automatic data analyses with optimized output make data analysis more efficient; users need significantly less user interaction compared to traditional analysis software, and the output is more comprehensive and still concise. This feature is essential for both experts and novices. 
Teaching statistics. Several features of the automatic analysis solution make this type of software advantageous for educational purposes. Students may rely on the task and on the conceptual understanding instead of the to-be-performed procedures when initiating an analysis, which makes the analysis more accessible and more understandable. Optimized output (e.g., graphical representation of the data, and organic structure of the result sections) provides explanation and context to the data. Moreover, various functions of the software and different sections of the output will help teachers to discuss essential topics and aspects of data analysis practices.

\section{General discussion}

Statistical data analysis practice faces several challenges, and many modifications have been recommended to remedy different aspects of the current analysis practice. Nonetheless, progress is slow, and there is still plenty of room for improvement. Software tools that make recommended procedures more accessible promote better data analysis practices (Wagenmakers et al., 2017).

In this paper, it was argued that automatic analysis and optimized output software can offer solutions for many aspects of these problems. Such a software can be faster, more precise, more efficient, more informative, and more accessible. These advantages can help to improve the current data analysis practice, mitigate the replication crisis, improve statistics education, and support methodological research.

The present work highlighted the advantages of an automatic data analysis solution and also discussed many potential problems with its use. Similar to other analysis software types, automatic data analysis is not a perfect solution for all circumstances. Rather, the general conditions where automatic analysis software can be a preferred solution were described here. Even so, it is the decision of researchers and students whether they will use an automatic analysis solution as their primary tool or as a supplementary tool.

While an initial set of principles for automatic analysis and optimized output was proposed here, by no means is this description complete or final. Additional ideas could extend or replace the present principles. Similarly, beyond the present examples, additional analyses could be added to the automatic analysis solutions. Many of these additional analyses will introduce challenges, such as what default priors should be used in Bayesian hypothesis testing (Simmons et al., 2011), and what default parameters should be used in data cleaning. Nonetheless, even in this early phase, automatic data analyses offers software solutions that are easier to use and that make the results more precise, more coherent, and more accessible compared to the usual software solutions.

\section{Acknowledgments}

The development of CogStat was supported by the Social Renewal Operational Program (TÁMOP) 4.1.2.A/1-11/1-2011-0018 and by the Content Pedagogy Research Program of the Hungarian Academy of Sciences: Extended Didactical Grant, 2016-2020; ID 471028. The writing of the present manuscript was supported by the mentioned Extended Didactical Grant and by Central 
European University, Hungary. I would like to thank Karolina Janacsek for her comments on an earlier version of the software. I thank all developers and localizers contributing to the code and the translation of the software, and I thank all the users, colleagues and students who gave advice on specific solutions used in CogStat (see a detailed list of contributors in the online documentation). I thank Petia Kojouharova, László Mérő, Dezső Németh, and András Vargha for their helpful comments on the manuscript.

\section{References}

Anscombe, F. J. (1973). Graphs in Statistical Analysis. The American Statistician, 27(1), 17-21. https://doi.org/10.1080/00031305.1973.10478966

Bakker, M., \& Wicherts, J. M. (2011). The (mis)reporting of statistical results in psychology journals. Behavior Research Methods, 43(3), 666-678. https://doi.org/10.3758/s13428-0110089-5

Beyth-Marom, R., Fidler, F., \& Cumming, G. (2008). Statistical Cognition: Towards EvidenceBased Practice in Statistics and Statistics Education. Statistics Education Research Journal, 7(2), 20-39.

Bowring, A., Maumet, C., \& Nichols, T. E. (2019). Exploring the impact of analysis software on task fMRI results. Human Brain Mapping, 40(11), 3362-3384.

https://doi.org/10.1002/hbm.24603

Brugman, S. (2021). Pandas-profiling [Jupyter Notebook]. pandas-profiling.

https://github.com/pandas-profiling/pandas-profiling (Original work published 2016)

Button, K. S., Ioannidis, J. P. A., Mokrysz, C., Nosek, B. A., Flint, J., Robinson, E. S. J., \& Munafò, M. R. (2013). Power failure: Why small sample size undermines the reliability of neuroscience. Nature Reviews Neuroscience, 14(5), 365-376.

https://doi.org/10.1038/nrn3475

Carp, J. (2012a). On the Plurality of (Methodological) Worlds: Estimating the Analytic Flexibility of fMRI Experiments. Frontiers in Neuroscience, 6.

https://doi.org/10.3389/fnins.2012.00149

Carp, J. (2012b). The secret lives of experiments: Methods reporting in the fMRI literature.

NeuroImage, 63(1), 289-300. https://doi.org/10.1016/j.neuroimage.2012.07.004 
Chambers, C. D. (2013). Registered Reports: A new publishing initiative at Cortex. Cortex, 49(3), 609-610. https://doi.org/10.1016/j.cortex.2012.12.016

Coulson, M., Healey, M., Fidler, F., \& Cumming, G. (2010). Confidence intervals permit, but don’t guarantee, better inference than statistical significance testing. Frontiers in Psychology, 1. https://doi.org/10.3389/fpsyg.2010.00026

Crawford, J. R., \& Howell, D. C. (1998). Comparing an Individual’s Test Score Against Norms Derived from Small Samples. The Clinical Neuropsychologist, 12(4), 482-486. https://doi.org/10.1076/clin.12.4.482.7241

Cumming, G. (2014). The New Statistics Why and How. Psychological Science, 25(1), 7-29. https://doi.org/10.1177/0956797613504966

Cumming, G., \& Finch, S. (2005). Inference by Eye: Confidence Intervals and How to Read Pictures of Data. American Psychologist, 60(2), 170-180.

Esteban, O., Markiewicz, C. J., Blair, R. W., Moodie, C. A., Isik, A. I., Erramuzpe, A., Kent, J. D., Goncalves, M., DuPre, E., Snyder, M., Oya, H., Ghosh, S. S., Wright, J., Durnez, J., Poldrack, R. A., \& Gorgolewski, K. J. (2019). fMRIPrep: A robust preprocessing pipeline for functional MRI. Nature Methods, 16(1), 111-116. https://doi.org/10.1038/s41592-0180235-4

Farrell, P. J., \& Rogers-Stewart, K. (2006). Comprehensive study of tests for normality and symmetry: Extending the Spiegelhalter test. Journal of Statistical Computation and Simulation, 76(9), 803-816. https://doi.org/10.1080/10629360500109023

Faul, F., Erdfelder, E., Lang, A.-G., \& Buchner, A. (2007). G*Power 3: A flexible statistical power analysis program for the social, behavioral, and biomedical sciences. Behavioral Research Methods, Instruments and Computers, 39, 175-191.

Fritz, C. O., Morris, P. E., \& Richler, J. J. (2012). Effect size estimates: Current use, calculations, and interpretation. Journal of Experimental Psychology. General, 141(1), 2-18. https://doi.org/10.1037/a0024338

GraphPad Software. (n.d.). GraphPad InStat. https://graphpad.com/scientific-software/instat/ 
Hoekstra, R., Kiers, H., \& Johnson, A. (2012). Are assumptions of well-known statistical techniques checked, and why (not)? Quantitative Psychology and Measurement, 3, 137. https://doi.org/10.3389/fpsyg.2012.00137

Hoekstra, R., Morey, R. D., Rouder, J. N., \& Wagenmakers, E.-J. (2014). Robust misinterpretation of confidence intervals. Psychonomic Bulletin \& Review, 21(5), 1157-1164. https://doi.org/10.3758/s13423-013-0572-3

Hunter, J. D. (2007). Matplotlib: A 2D graphics environment. Computing In Science \& Engineering, 9(3), 90-95.

Ioannidis, J. P. A. (2005). Why Most Published Research Findings Are False. PLoS Medicine, 2(8). https://doi.org/10.1371/journal.pmed.0020124

Kerr, N. L. (1998). HARKing: Hypothesizing After the Results are Known. Personality and Social Psychology Review, 2(3), 196-217. https://doi.org/10.1207/s15327957pspr0203_4

Killeen, P. R. (2005). An Alternative to Null-Hypothesis Significance Tests. Psychological Science, 16(5), 345-353. https://doi.org/10.1111/j.0956-7976.2005.01538.x

Krajcsi, A. (2021). Follow-up questions influence the measured number knowledge in the Give-anumber task. Cognitive Development, 57, 100968. https://doi.org/10.1016/j.cogdev.2020.100968

Leys, C., Ley, C., Klein, O., Bernard, P., \& Licata, L. (2013). Detecting outliers: Do not use standard deviation around the mean, use absolute deviation around the median. Journal of Experimental Social Psychology, 49(4), 764-766. https://doi.org/10.1016/j.jesp.2013.03.013

Maner, J. K. (2014). Let’s Put Our Money Where Our Mouth Is: If Authors Are to Change Their Ways, Reviewers (and Editors) Must Change With Them. Perspectives on Psychological Science, 9(3), 343-351. https://doi.org/10.1177/1745691614528215

Masicampo, E. J., \& Lalande, D. R. (2012). A peculiar prevalence of p values just below .05. The Quarterly Journal of Experimental Psychology, 65(11), 2271-2279. https://doi.org/10.1080/17470218.2012.711335

Matejka, J., \& Fitzmaurice, G. (2017). Same Stats, Different Graphs: Generating Datasets with Varied Appearance and Identical Statistics through Simulated Annealing. ACM SIGCHI 
Conference on Human Factors in Computing Systems. https://doi.org/10.1145/3025453.3025912

McKinney, W. (2010). Data Structures for Statistical Computing in Python. In S. van der Walt \& J. Millman (Eds.), Proceedings of the 9th Python in Science Conference (pp. 51-56).

Morey, R. D., Hoekstra, R., Rouder, J. N., Lee, M. D., \& Wagenmakers, E.-J. (2016). The fallacy of placing confidence in confidence intervals. Psychonomic Bulletin \& Review, 23(1), 103123. https://doi.org/10.3758/s13423-015-0947-8

Nickerson, R. S. (2000). Null hypothesis significance testing: A review of an old and continuing controversy. Psychological Methods, 5(2), 241-301. https://doi.org/10.1037/1082989X.5.2.241

Nosek, B. A., Spies, J. R., \& Motyl, M. (2012). Scientific Utopia: II. Restructuring Incentives and Practices to Promote Truth Over Publishability. Perspectives on Psychological Science. https://doi.org/10.1177/1745691612459058

Noughabi, H. A., \& Arghami, N. R. (2011). Monte Carlo comparison of seven normality tests. Journal of Statistical Computation and Simulation, 81(8), 965-972. https://doi.org/10.1080/00949650903580047

Oliphant, T. E. (2007). Python for Scientific Computing. Computing in Science \& Engineering, 9(3), 10-20. https://doi.org/10.1109/MCSE.2007.58

Open Science Collaboration. (2015). Estimating the reproducibility of psychological science. Science, 349(6251), aac4716. https://doi.org/10.1126/science.aac4716

Paton-Simpson, G. (n.d.). SOFA Statistics. http://www.sofastatistics.com/

Peterson, K., \& Caldwell, A. (2019). mbir: Magnitude-Based Inferences (1.3.5) [Computer software]. https://CRAN.R-project.org/package=mbir

Rochon, J., Gondan, M., \& Kieser, M. (2012). To test or not to test: Preliminary assessment of normality when comparing two independent samples. BMC Medical Research Methodology, 12(1), 81. https://doi.org/10.1186/1471-2288-12-81 
Romão, X., Delgado, R., \& Costa, A. (2010). An empirical power comparison of univariate goodness-of-fit tests for normality. Journal of Statistical Computation and Simulation, 80(5), 545-591. https://doi.org/10.1080/00949650902740824

Rosenthal, R. (1979). The file drawer problem and tolerance for null results. Psychological Bulletin, 86(3), 638-641. https://doi.org/10.1037/0033-2909.86.3.638

Schucany, W. R., \& Tony Ng, H. K. (2006). Preliminary Goodness-of-Fit Tests for Normality do not Validate the One-Sample Student t. Communications in Statistics - Theory and Methods, 35(12), 2275-2286. https://doi.org/10.1080/03610920600853308

Seabold, S., \& Perktold, J. (2010). Statsmodels: Econometric and statistical modeling with python. Proceedings of the 9th Python in Science Conference.

Silberzahn, R., Uhlmann, E. L., Martin, D. P., Anselmi, P., Aust, F., Awtrey, E., Bahník, Š., Bai, F., Bannard, C., Bonnier, E., Carlsson, R., Cheung, F., Christensen, G., Clay, R., Craig, M. A., Dalla Rosa, A., Dam, L., Evans, M. H., Flores Cervantes, I., ... Nosek, B. A. (2018). Many Analysts, One Data Set: Making Transparent How Variations in Analytic Choices Affect Results. Advances in Methods and Practices in Psychological Science, 1(3), 337-356. https://doi.org/10.1177/2515245917747646

Simmons, J. P., Nelson, L. D., \& Simonsohn, U. (2011). False-Positive Psychology: Undisclosed Flexibility in Data Collection and Analysis Allows Presenting Anything as Significant. Psychological Science, 22(11), 1359-1366. https://doi.org/10.1177/0956797611417632

The jamovi project. (2020). Jamovi (1.2) [Computer software]. https://www.jamovi.org Vallat, R. (2018). Pingouin: Statistics in Python. Journal of Open Source Software, 3(31), 1026. https://doi.org/10.21105/joss.01026

Vargha, A., Torma, B., \& Bergman, L. R. (2015). ROPstat: A General Statistical Package Useful for Conducting Person-Oriented Analyses. Journal for Person-Oriented Research, 1(1-2), 8798.

Wagenmakers, E.-J. (2007). A practical solution to the pervasive problems of p /values. Psychonomic Bulletin \& Review, 14(5), 779-804. https://doi.org/10.3758/BF03194105 
Wagenmakers, E.-J., Love, J., Marsman, M., Jamil, T., Ly, A., Verhagen, J., Selker, R., Gronau, Q. F., Dropmann, D., Boutin, B., Meerhoff, F., Knight, P., Raj, A., Kesteren, E.-J. van, Doorn, J. van, Šmíra, M., Epskamp, S., Etz, A., Matzke, D., ... Morey, R. D. (2017). Bayesian inference for psychology. Part II: Example applications with JASP. Psychonomic Bulletin \& Review, 1-19. https://doi.org/10.3758/s13423-017-1323-7

Wallace, B. C., Dahabreh, I. J., Trikalinos, T. A., Lau, J., Trow, P., \& Schmid, C. H. (2012). Closing the Gap between Methodologists and End-Users: R as a Computational Back-End. Journal of Statistical Software, 049(i05). http://econpapers.repec.org/article/jssjstsof/v_3a049_3ai05.htm

Weissgerber, T. L., Milic, N. M., Winham, S. J., \& Garovic, V. D. (2015). Beyond Bar and Line Graphs: Time for a New Data Presentation Paradigm. PLOS Biology, 13(4), e1002128. https://doi.org/10.1371/journal.pbio.1002128

Wicherts, J. M. (2017). The Weak Spots in Contemporary Science (and How to Fix Them). Animals, 7(12), 90. https://doi.org/10.3390/ani7120090

Wicherts, J. M., Bakker, M., \& Molenaar, D. (2011). Willingness to Share Research Data Is Related to the Strength of the Evidence and the Quality of Reporting of Statistical Results. PLOS ONE, 6(11), e26828. https://doi.org/10.1371/journal.pone.0026828

Wicherts, J. M., Borsboom, D., Kats, J., \& Molenaar, D. (2006). The Poor Availability of Psychological Research Data for Reanalysis. American Psychologist, 61(7), 726-728.

Wicherts, J. M., Veldkamp, C. L. S., Augusteijn, H. E. M., Bakker, M., van Aert, R. C. M., \& van Assen, M. A. L. M. (2016). Degrees of Freedom in Planning, Running, Analyzing, and Reporting Psychological Studies: A Checklist to Avoid p-Hacking. Frontiers in Psychology, 7. https://doi.org/10.3389/fpsyg.2016.01832

Wilkinson, L. (1999). Statistical methods in psychology journals: Guidelines and explanations. American Psychologist, 54(8), 594-604. https://doi.org/10.1037/0003-066X.54.8.594

Yap, B. W., \& Sim, C. H. (2011). Comparisons of various types of normality tests. Journal of Statistical Computation and Simulation, 81(12), 2141-2155. https://doi.org/10.1080/00949655.2010.520163 